\title{
Fully Private Auctions for the Highest Bid*
}

\author{
Jie Ma, Bin Qi, Kewei Lv \\ School of Cyber Security, University of Chinese Academy of Sciences, \\ State Key Laboratory of Information Security, Institute of Information Engineering, \\ Data Assurance and Communication Security Research Center, \\ Chinese Academy of Sciences, Beijing, 100093, P.R. China. \\ \{majie,qibin,lvkewei\}@iie.ac.cn
}

\begin{abstract}
We present a secure auction scheme with affordable computations and communication complexity. In this scheme, we use blockchain to publish commitment and some public messages only. All bidders are forced to perform protocol honestly and are not permitted to change or retract their bids. To preserve privacy, we use insulated ciphertext comparison protocol to compare the bids. Our scheme is more efficient than traditional auctions using MPC/ZKP primitives. The total number of interaction rounds for $N$ bidders is $3(N-1)$. Furthermore, when the comparisons is performed in parallel, the number of rounds dramatically decreases to $3 \log N$.
\end{abstract}

\section{CCS CONCEPTS}

- Applied computing $\rightarrow$ Online auctions.

\section{KEYWORDS}

Blockchain; Auction; Commitment; Integer Comparison Protocol

ACM Reference Format:

Jie Ma, Bin Qi, Kewei Lv. 2019. Fully Private Auctions for the Highest Bid. In ACM Turing Celebration Conference - China (ACM TURC 2019) (ACM TURC 2019), May 17-19, 2019, Chengdu, China. ACM, New York, NY, USA, 6 pages. https: //doi.org/10.1145/3321408.3321570

\section{INTRODUCTION}

With the need of electronic commerce, online auctions have gained much attention as the major phenomenon. Privacy plays an important role in auction design, and many schemes have been proposed to not only preserve the privacy of sealed

\footnotetext{
* This work is partially supported by National Key R\&D Program of China(no. 2017YFB0802500), The 13th Five-Year National Cryptographic Development Foundation(no. MMJJ20180208), Beijing Science and Technology Commission (no. Z181100002718001) and NSF (no. $61272039)$.

Permission to make digital or hard copies of all or part of this work for personal or classroom use is granted without fee provided that copies are not made or distributed for profit or commercial advantage and that copies bear this notice and the full citation on the first page. Copyrights for components of this work owned by others than ACM must be honored. Abstracting with credit is permitted. To copy otherwise, or republish, to post on servers or to redistribute to lists, requires prior specific permission and/or a fee. Request permissions from permissions@acm.org.

ACM TURC 2019, May 17-19, 2019, Chengdu, China

(C) 2019 Association for Computing Machinery.

ACM ISBN 978-1-4503-7158-2/19/05 . .\$15.00

https://doi.org/10.1145/3321408.3321570
}

bid, but also determine the highest bid. We do with the case, where one auctioneer and $N$ bidders want to make an agreement on the highest price of a good but each bidder will not reveal his private bid. Bidders submit bid and hope to win the auction for the highest price that can be determined by a public rule.

[11] shows four types of auctions. For English auction, bidders increasingly submit higher bids until they are not willing to pay more than the current highest bid. During the process, every bidder does not wish to reveal his value. If the bidder directly shows the bid to the auctioneer, this may cause unfairness since the auctioneer may leak this bid value to other bidders. Differently, for Vickrey auctions and First-price sealed-bid auctions, bidders can submit their bids in sealed envelopes and hand them to the auctioneer. Subsequently, the auctioneer opens the envelopes to determine the winner. The advantage of the sealed-bid auctions is that no bidder learns any information about the other bids, so the bidders are encouraged to bid according to their monetary valuation.

Joint bidding [7] is a form of alliance and is used extensively in the construction and insurance industries, where firms often find themselves unable to bid for large projects individually. Joint bidding allows a group of firms to collectively undertake a big project beyond the reach of any single firm in the group. The logic is to take advantage of the collective size of the group. Thus, this type of alliance has more of a collective focus than an individual one. To choose proper partners, the project owner need to check their financial assets which they do not like to uncover. This occasion can be seen as auction, through which, the project owner can select some candidates by selecting more than one winners for further consideration.

In this paper, we adopt blockchain to publish some public messages only, and every bidder commits to his bid with some deposit on it. Thus, bids can not be changed or revoked, and bidders are encouraged to perform correctly. The aborting bidders will be financially penalized because of their attempt to deviate from the protocol and prematurely abort to affect the behavior of the auction protocol including making false bids and wrong complaint. To preserve bid privacy, each bidder executes the insulated integer comparison protocol (ICP) off-chain to compare his bid with another under ciphertext, that is, a same bid in different ICP is encrypted to different ciphertext. A winner will be determined according to the highest bid, who is supposed to open his commitment onto blockchain. If a bidder has a complaint, then he can broadcast the complaint on-chain, and the auctioneer will broadcast 
the complaint result on blockchain after re-executing ICP. Since the original ICP is not practical enough to achieve a real auction, we extend it to compare larger integers by separately comparing corresponding coefficients. At last, we apply our scheme to joint biding to select proper partners.

Our schemes need $3(N-1)$ interaction rounds for $N$ bidders in total since they only perform $(N-1)$ pairwise three-round comparisons. When a binary tree structure is adopted to make pairwise comparisons, the number of rounds can be reduced to $3 \log N$. Indeed, we regard all $N$ bidders as leaf nodes of a tree at the bottom layer, and the auctioneer $\mathbf{A}$ divides all bidders into $\lfloor N / 2\rfloor$ pairs to run ICP respectively in parallel getting the $\lfloor N / 2\rfloor$ higher bids which can be considered as parent nodes of leaves, and then they are divided into $\lfloor N / 4\rfloor$ pairs to run pairwise ICP in parallel and so on. A recursively divides the nodes at lower layers into pairs to perform ICPs obtaining the nodes at upper layers. All the bids of parent nodes are larger than the bids of their children, and the root will hold the highest bid among all bidders. Since ICPs at the same layer can be executed in parallel in 3 rounds, the total rounds will be $3 \log N$. In fact, ICP can be done in 2 rounds by adding the messages transferred in the first round to the commitment and broadcasting them on blockchain.

This paper is organized as follows. In section 1, we present an introduction. The related researches are given in section 2 . In Section 3, we give a description for background including the adversary model together with the two cryptographic primitives: the commitment scheme and the secure integer comparison protocol. In Section 4, we present our main auction scheme and the parallelized version. In Section 5, we extend the comparison protocol as well as the auction scheme, and a conclusion is given in Section 6 .

\section{RELATED WORK}

There have been plenty of researches on auction [5, 9], focusing on combining cryptocurrencies with secure multiparty computation protocols (MPC) $[2,14]$ and/or zero-knowledge proofs $(Z K P)[1,3,12,13]$. Typically, the cryptocurrency is used to guarantee fairness and correctness, and avoid deviations from the MPC or ZKP protocol while MPC and/or ZKP are used to determine a winner correctly with public verifiability.

Kosba et al. [10] presented Hawk to implement the auction using secure MPC on the blockchain. The security of a Hawk program is guaranteed to satisfy on-chain privacy that protects the flow of money and data from the public view, and contractual security that protects the parties in the agreement of the contract from each other. Galal et al. [8] proposed the verifiable sealed-bid auction on top of blockchain by smart contract via homomorphic commitment and public key encryption scheme. The correctness is verified by interactive ZKP protocols between the smart contract and the auctioneer. Blass and Kerschbaum [4] proposed Strain a protocol to implement sealed-bid auctions on top of blockchains that protects the bid privacy against fully-malicious parties. They used a two-party comparison mechanism to replace MPC primitives, which achieved improved efficiency. The outcome of the comparison can be verified by ZKP. An additional ZKP protocol is used to verify that the comparisons only involved the committed bids. Moreover, the authors mentioned that the proposed protocol leaks the order of bids. Sánchez [15] presented Raziel, a system that combines MPC and proof-carrying code to guarantee the privacy, correctness and verifiability of smart contracts. They used ZKPs to show the validity of smart contracts to third parties. Moreover, the author also proposed an incentive-based scheme for miners to generate preprocessed data of MPC.

While these studies above all use MPC and/or ZKP primitives, which need more interactions and are quite inefficient, in our scheme, we use an insulated integer comparison protocol to determine the winner under ciphertext and a commitment scheme with deposit, which are much more simple and efficient.

\section{PRELIMINARIES}

In this section, we present the adversary model and security definition, together with the two primitives used in our auction scheme.

Convention. We say someone broadcasts something mean$\mathrm{s}$ that he publishes his message on the blockchain via a transaction. We denote by $\lambda$ as the security parameter for the cryptosystems. We write $x \leftarrow_{\$} \mathcal{X}$ meaning that $x$ is uniformly and independently chosen from the set $\mathcal{X}$.

\subsection{Adversary Model}

All bidders and the adversaries run in polynomial time (PT). We consider two adversaries $\mathcal{A}_{1}$ and $\mathcal{A}_{2}$ who have different capabilities, are non-colluding, and corrupt different parties. $\mathcal{A}_{1}$ can adaptively corrupt one or more bidders such that: 1 ) the corrupted bidders perform protocol correctly but attempt to obtain others' privacy. 2) any corrupted bidder may make a false bid, that is, what she commits to does not agree with what she uses in the comparison protocol. 3) any corrupted bidder can submit a complaint about the result of auction at any time. $\mathcal{A}_{2}$ is passive, who may control the auctioneer with no active behaviors but to obtain others' privacy. In addition, any participants can read and publish messages on blockchain. We assume the communication channel between participants is authenticated.

Let $\mathcal{P}$ be a set of parties, who perform a protocol $\Pi$ to compute a function $F$ given inputs $I n_{\mathcal{P}}$ and output $O u t_{\mathcal{P}}$. For a corrupted subset $I$ of $\mathcal{P}(I \subseteq \mathcal{P})$, the view of $I$ is denoted by $\mathbf{V I E W}_{I}^{\Pi}\left(\operatorname{In}_{\mathcal{P}}, O u t_{I}, \phi_{I}\right)$, where $\phi_{I}$ contains messages and random numbers selected by $P_{i, i \in I}$ and related information during running $\Pi$. Let $\operatorname{Sim}_{I}$ be a simulator that takes the inputs of all $P_{i, i \in I}\left(I n_{I}\right)$ and the outcome of $F$ received by $P_{i}\left(\right.$ Out $\left._{I}\right)$ to produce a transcript of the protocol.

Definition 3.1. A protocol $\Pi$ is secure against adversaries $\mathcal{A}_{1}$ and $\mathcal{A}_{2}$ if there exists a probabilistic polynomial time (PPT) simulator $\mathbf{S i m}_{I}$ such that the distributions, $\mathbf{S i m}_{I}$ and 
VIEW $_{I}^{\Pi}$, are computationally indistinguishable (for brevity by symbol $\left.\approx_{c}\right)$, that is, $\operatorname{Sim}_{I}\left(\operatorname{In}_{I}, F\left(I n_{\mathcal{P}}\right), O u t_{I}, \phi_{I}\right) \approx_{c}$ $\operatorname{VIEW}_{I}^{\Pi}\left(\operatorname{In}_{\mathcal{P}}, F\left(\operatorname{In}_{\mathcal{P}}\right)\right.$, Out $\left._{I}, \phi_{I}\right)$.

\subsection{Commitment Scheme with Deposit}

As one of the applications of blockchain, [1] presents a blockchain-based commitment scheme (CS) with deposit. In our scheme, we use it to bind every bidder with his bid, and force him to obey the protocol.

At pre-condition phrase, committer $\mathbf{C}$ and recipient $\mathbf{R}$ agree on a hash function $\mathcal{H}$ and the deposit value $d$ which can only be redeemed by $\mathbf{C}$. At commitment phase, $\mathbf{C}$ computes $h=\mathcal{H}(s \| y)$ as a commitment to $y$ together with some randomness $s$, and broadcasts it on blockchain by a transaction CS.Commit(y). Then, $\mathbf{C}$ creates a transaction CS.Fuse, sends it with his signature to $\mathbf{R}$, which enables $\mathbf{R}$ to claim the deposit of $\mathbf{C}$ if $\mathbf{C}$ refuses to open the commitment correctly. At opening phase, $\mathbf{C}$ broadcasts transaction $C S$.Open revealing the string $s \| y$ to redeem his deposit. If CS.Open does not appear on the blockchain after a deadline, then $\mathbf{R}$ broadcasts CS.Fuse to gain deposit of $\mathbf{C}$.

Obviously, honest committer will not lose his deposit. If committer refuses to open his commitment correctly, then the recipient could claim C's deposit. The binding and hiding properties are guaranteed by collision resistance and unpredictability property of hash function.

\subsection{Integer Comparison Protocol}

Now, we give a simple description about the integer comparison protocol (ICP) [6], which consists of two semantically secure public key encryption (PKE) schemes, $P K E_{1}$ and $P K E_{2}$.

For $P K E_{1}=(\mathbf{K G e n}$, Enc, Dec $)$. First, run $\mathbf{K G e n}\left(1^{\lambda}\right)$ to generate public key $\mathcal{P K}=\left(n, b, d_{M}, g, h, u\right)$ and private key $\mathcal{S K}=(x)$, where $n=p q, p=2 b^{d_{M}} p_{s} p_{t}+1, q=2 b^{d_{M}} q_{s} q_{t}+$ $1, h^{p_{s} q_{s}}=1 \bmod n, x=p_{s} q_{s} x^{\prime}, x^{\prime}=\left(p_{s} q_{s}\right)^{-1} \bmod b^{d_{M}}, p_{s}$ and $q_{s}$ are independently and randomly chosen $u$-bit primes such that computing the discrete logarithm is computationally infeasible. To encrypt message $m \in\left\{0, \ldots, d_{M}-1\right\}$ given $\mathcal{P K}$, pick random $r \leftarrow_{\$}\left\{1, \ldots, 2^{u}-1\right\}$ and compute ciphertext $C=g^{b^{m}} h^{r} \bmod n$. To decrypt a ciphertext $C$ given private key $\mathcal{S} \mathcal{K}$, compute $C^{x} \bmod n=g^{b^{m}}$, and $m$ can be recovered efficiently since $g$ and $b$ are chosen to be small. $P K E_{1}$ satisfies that if $m_{1}+m_{2}<d_{M}$, then $\mathbf{E n c}_{1}\left(m_{1}\right)^{b^{m_{2}}}=\mathbf{E n c}_{1}\left(m_{1}+m_{2}\right)$, otherwise, $\mathbf{E n c}_{1}\left(m_{1}\right)^{b^{m_{2}}}=\mathbf{E n c}_{1}(0)$. It is semantically secure under the Small RSA Subgroup Decision (for brevity, sRSA-SD) Assumption as following shown.

Definition 3.2. (sRSA-SD Assumption) Given an RSA quintuple $\left(n, b, d_{M}, g, u\right)$ and $x \in Q R_{n}$, we say it satisfies the sRSA-SD Assumption if for any polynomial time algorithm $\mathcal{B}$, the advantage of $\mathcal{B}$ in deciding whether $x$ has order $p_{s} q_{s}$ or not is negligible.

$P K E_{2}=\left(\mathbf{G e n}_{\oplus}, \mathbf{E n c}_{\oplus}\right.$, Dec $\left._{\oplus}\right)$ [16] with message space $\mathcal{M}_{\oplus}$ is additively homomorphic and used to test equality.
Specifically, the ICP is executed by two parties, $P_{1}$ and $P_{2}$, holding $m_{1}$ and $m_{2}\left(0 \leq m_{1}, m_{2}<d_{M}\right)$ respectively, who try to determine which one of $m_{1}$ and $m_{2}$ is larger without uncovering them.

(1) $P_{1}$ runs $\operatorname{KGen}\left(1^{\lambda}\right)$ getting the key pair $\left(\mathcal{P} \mathcal{K}_{1}, \mathcal{S} \mathcal{K}_{1}\right)$, he publishes his public key $\mathcal{P} \mathcal{K}_{1}=\left(n, b, d_{M}, g, h, u\right)$ and keeps the private key $\mathcal{S} \mathcal{K}_{1}=(x)$ as secret; $P_{2}$ runs $\operatorname{Gen}_{\oplus}\left(1^{\lambda}\right)$ obtaining the key pair $\left(\mathcal{P} \mathcal{K}_{2}, \mathcal{S} \mathcal{K}_{2}\right)$, she publishes her public key $\mathcal{P} \mathcal{K}_{2}$ holding the corresponding private key $\mathcal{S} \mathcal{K}_{2}$.

(2) $P_{1}$ selects $r_{1} \leftarrow \$\left\{1, \ldots, 2^{u}-1\right\}$ and computes $C=$ $g^{b^{m_{1}}} h^{r_{1}}$, then he sends $C$ to $P_{2}$.

(3) $P_{2}$ selects $r_{2} \leftarrow \$\left\{1, \ldots, 2^{u}-1\right\}, s \leftarrow \$\left\{1, \ldots, b^{d_{M}}-1\right\}$, s.t., $s \not \equiv 0 \bmod b$, computes $D=C^{b^{\left(d_{M}-m_{2}\right)}} g^{s} h^{r_{2}}, A_{2}=$ Enc $_{\oplus}(s)$, then she sends $\left(D, A_{2}\right)$ to $P_{1}$.

(4) $P_{1}$ computes $g^{\omega}=D^{x}$, solves $\omega=\log _{g}\left(g^{\omega}\right)$, computes $A_{1}^{\prime}=\mathbf{E n c}_{\oplus}(-w) \cdot A_{2}$, then blinds $A_{1}^{\prime}$ by computing $A_{1}=$ $\left(A_{1}^{\prime}\right)^{r}$ for $0 \neq r \leftarrow_{\$} \mathcal{M}_{\oplus}$, Afterwards he sends $A_{1}$ to $P_{2}$.

(5) $P_{2}$ computes $m=\operatorname{Dec}_{\oplus}\left(A_{1}\right)$. If $m=0, P_{2}$ outputs True $\left(\right.$ i.e. $\left.m_{1} \geq m_{2}\right)$, and False otherwise.

We present the following Lemma from [6].

LEMMA 3.3. The ICP above protects the privacy of both $P_{1}$ and $P_{2}$ under $s R S A-S D$ Assumption.

\section{FULLY PRIVATE AUCTION SCHEME}

In this part, we present our auction scheme in sequential and parallel modes respectively. In order to avoid making a false bid, we use the commitment scheme (CS) requiring every bidder to bind his bid and submit some deposit. The deposit can not be redeemed as a possible punishment if the agreement of his bid is inconsistent or a wrong complaint is given. To preserve privacy, a secure integer comparison protocol (ICP) is used to compare the bids in ciphertexts.

\subsection{Sequential Scheme}

In an auction, the auctioneer wants to auction some target evaluated commodity, and bidders submit their own bids but do not want to reveal their bids to anyone. So the auctioneer has to determine a winner in the case of unknowing the bids. Usually, bidders submit their bids in sealed envelopes, afterwards, the auctioneer opens them secretly and determines the highest value as the winner. This leads to information leakage since the auctioneer knows all bids, and bidders even can reject to complete the duties honestly.

To overcome this gap, the auctioneer $\mathbf{A}$ first evaluates the target value $x$ and commits to it via CS with some deposit then publishes it. Let $y_{h}$ from $\mathbf{B}_{\mathbf{h}}$ be the current highest bid value and initially, $y_{h} \leftarrow x$ from $\mathbf{A}$. In every round of comparison, a bidder $\mathbf{B}_{\mathbf{i}}$ biding $y_{i}$, commits to $y_{i}$ via CS with some deposit by broadcasting the transaction CS.Commit $\left(y_{i}\right)$ onto blockchain and sending CS.Fuse to A. Then, $\mathbf{B}_{\mathbf{i}}$ runs ICP with $\mathbf{B}_{\mathbf{h}}$ to compare $y_{i}$ with $y_{h}$, after which, $\mathbf{B}_{\mathbf{i}}$ learns the comparison result and sends the result to $\mathbf{A}$. If $y_{i}>y_{h}, \mathbf{A}$ updates $y_{h}$ to be $y_{i}$ and $\mathbf{B}_{\mathbf{i}}$ plays the role of $\mathbf{B}_{\mathbf{h}}$ in the next comparison. Otherwise, $\mathbf{A}$ selects 
another bidder $\mathbf{B}_{\mathbf{i}+\mathbf{1}}$ to repeat the process above. At the end, a winner $\mathbf{B}_{\mathbf{w}}$ is determined according to the last $y_{h}$. During the process, if someone submits a complaint, then he is required to send his bid to $\mathbf{A}$, who runs ICP with the complained bidder and broadcasts the result of comparison on the complaint without revealing the related bids.

As for deposit, each failed bidder can broadcasts CS.Open onto blockchain to redeem his deposit. But CS.Open will reveal the bid privacy. Here, we allow each bidder to redeem his deposit by a transaction CS.Refund which is valid only when it is signed by both the bidder and the auctioneer. At last, the winner should open his commitment onto blockchain for public verification. If a bidder submits a wrong complaint or performs dishonestly affecting the auction, then he cannot redeem his deposit since $\mathbf{A}$ will broadcast CS.Fuse to punish him.

We present the specific description in the following. The deposit value and the involved hash function can be determined by all bidders in advance.

(1) A first commits to $x$ with some deposit and broadcasts the commitment and $x$ onto the blockchain. Every bidder $\mathbf{B}_{\mathbf{i}}$ runs CS to commit $y_{i}$ with some deposit and broadcasts CS.Commit C $_{i}$ together with the related public keys. Then he sends CS.Fuse C $_{i}$ to A.

(2) $\mathbf{A}$ selects $\mathbf{B}_{\mathbf{i}}$ to run ICP with $\mathbf{B}_{\mathbf{h}}$ to compare $y_{i}$ with $y_{h}$, where $\mathbf{B}_{\mathbf{h}}$ plays the role of $P_{1}$ while $\mathbf{B}_{\mathbf{i}}$ acts as $P_{2} . \mathbf{B}_{\mathbf{i}}$ then gets the comparison result and sends this result to $\mathbf{A}$. If $y_{i}>y_{h}$, $\mathbf{A}$ (of course does not know the values) updates $\left(y_{h}, \mathbf{B}_{\mathbf{h}}\right)$ by $\left(y_{i}, \mathbf{B}_{\mathbf{i}}\right)$. Otherwise, $y_{h}$ will not be changed in the next round comparison. Then, $\mathbf{A}$ selects another bidder $\mathbf{B}_{\mathbf{i}+\mathbf{1}}$ to execute ICP with $\mathbf{B}_{\mathbf{h}}$. The loser can broadcast transaction CS.Open or create a transaction CS.Refund with his signature and sends it to $\mathbf{A}$.

(3) Repeat step 2 until a winner $\mathbf{B}_{\mathbf{w}}$ is determined, who should open his commitment for verification. During the process, if there is a complaint occurring, then the complainer is required to send his bid to $\mathbf{A}$, who runs ICP with the complained bidder and broadcasts the result of comparison on the complaint without revealing the related bids. If a complainer fails or a bidder performs dishonestly affecting the auction, then he cannot redeem his deposit since $\mathbf{A}$ will broadcast CS.Fuse to punish him. Finally, A broadcasts all received $C S$.Refunds after signing. Honest bidders can now redeem their deposit.

In this scheme, A finally reveals the bid $y_{w}$ to the public, in fact, it is not necessary, since every bidder with doubts can re-compare his bid with $y_{w}$ before $y_{w}$ is uncovered to $\mathbf{A}$ Our scheme determines one winner if there exist more than one bidders holding the same highest bid, since $y_{h}$ is updated only when the new chosen bid by $\mathbf{A}$ is larger than it. In step 2 , at the end of each ICP, we require the loser to send the result to $\mathbf{A}$, otherwise, he will not get the signature of $\mathbf{A}$ for his CS.Refund. The winner is incentivised to cooperate but it will be regarded as self abstention if he does not reply. Moreover, our auction scheme always determines the winner with the highest bid, and the total number of interaction rounds for $N$ bidders is $3(N-1)$ since we need to perform
$(N-1)$ pairwise comparisons. In fact, ICP can be done in 2 rounds by adding the messages transferred in the first round to the commitment and broadcasted on blockchain which can be read by any bidder.

TheOREm 4.1. Under sRSA-SD Assumption, our scheme protects the bid privacy of all bidders. Moreover, honest bidders can redeem their deposit.

Sketch of Proof. Obviously, all honest bidders will not lose their deposit. As for bid privacy, every bidder broadcasts his commitment to his bid onto the blockchain, and the adversaries can read it but get no help from the information on the blockchain. In the comparison phase, $\mathbf{A}$ just knows the comparison result, and honest bidders will not submit complaint, and their bid privacy will not be leaked even $\mathcal{A}_{2}$ corrupts A. If $\mathcal{A}_{1}$ submits a complaint, he will not get the bid privacy of the counterpart from $\mathbf{A}$ except for the result of running ICP between $\mathbf{A}$ and the bidder complained.

If $\mathcal{A}_{1}$ adaptively corrupts only one bidder. In this case, the corrupted bidder involves in some execution of ICP. $\mathcal{A}_{1}$ could not obtain the privacy of others by the security of ICP. Otherwise, $\mathcal{A}_{1}$ adaptively corrupts more than one bidders to run more ICPs. In each ICP, $\mathcal{A}_{1}$ at most learns the comparison result other than the bid. However, $\mathcal{A}_{1}$ may adaptively run many ICPs with a same honest bidder $\mathbf{B}_{0}$ holding $y_{0}$ to gain the interval of $y_{0}$, but $y_{0}$ is still preserved.

Since the channel is authenticated and all messages communicated are ciphertexts. The bid privacy of any bidder is preserved in the communication.

\subsection{Parallelized Scheme}

To achieve parallelized executions, we regard all $N$ bidders as leaf nodes in a binary tree at the bottom layer. In the first time, the auctioneer $\mathbf{A}$ divides all bidders into $\lfloor N / 2\rfloor$ pairs, every pair at the same layer perform ICP in parallel, and the larger bids are set to be the parent nodes. If some leaf can not be matched with another since $N$ may be an odd, then it is considered as a parent node. In the following procedure, we do with the nodes in the same ways, which could not be matched at their layer. A then divides the $\lfloor N / 2\rfloor$ parent nodes into $\lfloor N / 4\rfloor$ pairs in the second time to run pairwise ICP simultaneously and so on. A recursively divides the nodes at lower layers into pairs to perform ICPs obtaining the nodes at upper layers. All the bids of parent nodes are no less than the bids of their children, and the root will hold the largest bid. Since ICPs on the same layer can be executed in parallel in 3 rounds, then the total rounds will be $3 \log N$.

\section{EXTENSION}

Usually, $d_{M}$ in $P K E_{1}$ satisfies $b^{d_{M}}<\frac{1}{4}|n|-\lambda$, which is too small to be practical. So we extend ICP to compare larger integers.

\subsection{Extended ICP}

For $\ell$-bit integers larger than $d_{M}, \beta$ and $\alpha$, held by $P_{1}$ and $P_{2}$, respectively, we can represent them in base $d_{M}$, and perform 
ICP to compare corresponding coefficients separately. Let $k=$ $\left\lceil\log _{d_{M}}\left(2^{\ell}\right)\right\rceil$, then $\beta=\beta_{k-1} d_{M}^{k-1}+\beta_{k-2} d_{M}^{k-2}+\cdots+\beta_{1} d_{M}+\beta_{0}$ and $\alpha=\alpha_{k-1} d_{M}^{k-1}+\alpha_{k-2} d_{M}^{k-2}+\cdots+\alpha_{1} d_{M}+\alpha_{0}$, where $0 \leq \alpha_{i}, \beta_{i}<d_{M}$. If $\alpha>\beta$ then exactly one of the $k$ Boolean expressions below is True: $\left(\alpha_{k-1}>\beta_{k-1}\right),\left(\alpha_{k-1}=\beta_{k-1}\right) \wedge$ $\left(\alpha_{k-2}>\beta_{k-2}\right),\left(\alpha_{k-1}=\beta_{k-1}\right) \wedge\left(\alpha_{k-2}=\beta_{k-2}\right) \wedge\left(\alpha_{k-3}>\right.$ $\left.\beta_{k-3}\right), \cdots,\left(\alpha_{k-1}=\beta_{k-1}\right) \wedge\left(\alpha_{k-2}=\beta_{k-2}\right) \wedge \cdots \wedge\left(\alpha_{0}>\beta_{0}\right)$.

Since ICP only determines $\alpha_{j} \geq \beta_{j}$, we do not know whether $\beta_{j}=\alpha_{j}$ or not. To do with this, we run ICP twice to check $\left.\left(\alpha_{j} \geq \beta_{j}\right) \wedge\left(\alpha_{j} \geq \beta_{j}+1\right)\right)$ or not. If yes, then $\alpha_{j}>\beta_{j}$, otherwise, $\alpha_{j}=\beta_{j}$.

The extension denoted by eICP to compare $\alpha$ with $\beta$, is shown in the following, where $p_{0}$ is a public prime larger than $d_{M}$.

(1) $P_{1}$ runs $\operatorname{KGen}\left(1^{\lambda}\right)$ to generate key pair $\left(\mathcal{P} \mathcal{K}_{1}, \mathcal{S} \mathcal{K}_{1}\right)$, where $\mathcal{S} \mathcal{K}_{1}$ is private and $\mathcal{P} \mathcal{K}_{1}=\left(n, b, d_{M}, g, h, u\right)$ is public; $P_{2}$ runs $\operatorname{Gen}_{\oplus}\left(1^{\lambda}\right)$ to obtain her key pair $\left(\mathcal{P K} \mathcal{K}_{2}, \mathcal{S} \mathcal{K}_{2}\right)$. To compare $\alpha$ with $\beta$, both are expressed into $d_{M}$-ary with coefficients $\alpha_{j}$ and $\beta_{j}$ as above. Then all participants perform the following, where $j=k-1, \ldots, 0$.

(2) $P_{1}$ selects $r_{1, j}^{1}, r_{1, j}^{2} \leftarrow_{\$}\left\{1, \ldots, 2^{u}-1\right\}$, and computes $C_{j}^{1}=g^{b^{\left(d_{M}-\beta_{j}\right)}} h^{r_{1, j}^{1}}, C_{j}^{2}=g^{b^{\left(d_{M}-\beta_{j}-1\right)}} h^{r_{1, j}^{2}}$. Then he send$\mathrm{s}\left(C^{1}, C^{2}\right)$ to $P_{2}$, where $C^{1}=\left(C_{k-1}^{1}, \ldots, C_{0}^{1}\right)$ and $C^{2}=$ $\left(C_{k-1}^{2}, \ldots, C_{0}^{2}\right)$.

(3) $P_{2}$ selects $r_{2, j}^{1}, r_{2, j}^{2} \leftarrow \$\left\{1, \ldots, 2^{u}-1\right\}$ and $s_{j}^{1}, s_{j}^{2} \leftarrow \$$ $\left\{1, \ldots, b^{d_{M}}-1\right\}$, s.t., $s_{j}^{1}, s_{j}^{2} \not \equiv 0 \bmod b$. She computes $A_{2, j}^{1}=$ $\operatorname{Enc}_{\oplus}\left(s_{j}^{1}\right), A_{2, j}^{2}=\mathbf{E n c}_{\oplus}\left(s_{j}^{2}\right), D_{j}^{1}=\left(C_{j}^{1}\right)^{b^{\alpha_{j}}} g^{s_{j}^{1}} \cdot h^{r_{2, j}^{1}}, D_{j}^{2}=$ $\left(C_{j}^{2}\right)^{b^{\alpha_{j}}} g^{s_{j}^{2}} h^{r_{2, j}^{2}},, B_{2, k-1}=\mathbf{E n c}_{\oplus}(0), B_{2, k-2}=\mathbf{E n c}_{\oplus}\left(p_{0}^{k-1}\right.$. $\left.\alpha_{k-1}\right), B_{2, k-3}=\mathbf{E n c}_{\oplus}\left(p_{0}^{k-2} \cdot \alpha_{k-2}\right), \cdots, B_{2,0}=\mathbf{E n c}_{\oplus}\left(p_{0}\right.$. $\left.\alpha_{1}\right)$. Then she sends $\left(D^{1}, D^{2}, A_{2}^{1}, A_{2}^{2}, B_{2}\right)$ to $P_{1}$, where $D^{1}=$ $\left(D_{k-1}^{1}, \ldots, D_{0}^{1}\right), D^{2}=\left(D_{k-1}^{2}, \ldots, D_{0}^{2}\right), A_{2}^{1}=\left(A_{2, k-1}^{1}, \ldots\right.$, $\left.A_{2,0}^{1}\right), A_{2}^{2}=\left(A_{2, k-1}^{2}, \ldots, A_{2,0}^{2}\right), B_{2}=\left(B_{2, k-1}, \ldots, B_{2,0}\right)$.

(4) $P_{1}$ gets the quintuple $\left(D^{1}, D^{2}, A_{2}^{1}, A_{2}^{2}, B_{2}\right)$, computes $w_{j}^{1}=\operatorname{Dec}\left(D_{j}^{1}\right), w_{j}^{2}=\operatorname{Dec}\left(D_{j}^{2}\right), A_{1, j}^{1}=\operatorname{Enc}_{\oplus}\left(-w_{j}^{1}\right) \cdot A_{2, j}^{1}$, $A_{1, j}^{2}=\mathbf{E n c}_{\oplus}\left(-w_{j}^{2}\right) \cdot A_{2, j}^{2}$, and $B_{1, k-1}=\mathbf{E n c}_{\oplus}(0) \cdot B_{2, k-1}$, $B_{1, k-2}=\operatorname{Enc}_{\oplus}\left(-p_{0}^{k-1} \cdot \beta_{k-1}\right) \cdot B_{2, k-2} \cdot B_{1, k-1}, B_{1, k-3}=$ $\mathbf{E n c}_{\oplus}\left(-p_{0}^{k-2} \cdot \beta_{k-2}\right) \cdot B_{2, k-3} \cdot B_{1, k-2}, \cdots, B_{1,0}=\mathbf{E n c}_{\oplus}\left(-p_{0}\right.$. $\left.\beta_{1}\right) \cdot B_{2,0} \cdot B_{1,1}$. Then he computes $A_{j}^{\prime}=\left[A_{1, j}^{1} \cdot A_{1, j}^{2} \cdot B_{1, j}\right]^{r_{j}^{B}}$, where $0 \neq r_{j}^{B} \leftarrow \$ \mathcal{M}_{\oplus}$. He shuffles $A^{\prime}=\left(A_{k-1}^{\prime}, \ldots, A_{0}^{\prime}\right)$ to get $A=\left(A_{k-1}, \ldots, A_{0}\right)$ by a random permutation $\pi$, and sends $A$ to $P_{2}$. (5) $P_{2}$ receiving $A=\left(A_{k-1}, \ldots, A_{0}\right)$, computes $m_{j}=\operatorname{Dec}_{\oplus}\left(A_{j}\right)$. If there exist only one $j$, s.t. $m_{j}=0$, outputs True (i.e., $\alpha>\beta$ ), otherwise, outputs False (i.e., $\alpha \leq \beta$ ).

Correctness. First, for $w_{j}, w_{j}^{1}=b^{d_{M}-\beta_{j}+\alpha_{j}}+s_{j}^{1}$ and $w_{j}^{2}=b^{d_{M}-\beta_{j}-1+\alpha_{j}}+s_{j}^{2}$. If $\alpha_{j}>\beta_{j}$, then $\left(\alpha_{j} \geq \beta_{j}\right) \wedge\left(\alpha_{j} \geq\right.$ $\left.\beta_{j}+1\right)$, and $\left(w_{j}^{1}=s_{j}^{1}\right) \wedge\left(w_{j}^{2}=s_{j}^{2}\right)$, thus $A_{1, j}^{1} \cdot A_{1, j}^{2}=$ Enc $_{\oplus}\left(-w_{j}^{1}+s_{j}^{1}+-w_{j}^{2}+s_{j}^{2}\right)=$ Enc $_{\oplus}(0)$. If $\alpha_{j}=\beta_{j}$, then $\left(\alpha_{j} \geq \beta_{j}\right) \wedge\left(\alpha_{j}<\beta_{j}+1\right)$, and $\left(w_{j}^{1}=s_{j}^{1}\right) \wedge\left(w_{j}^{2} \neq s_{j}^{2}\right) ;$ If $\alpha_{j}<$ $\beta_{j}$, then $\left(\alpha_{j}<\beta_{j}\right) \wedge\left(\alpha_{j}<\beta_{j}+1\right)$, and $\left(w_{j}^{1} \neq s_{j}^{1}\right) \wedge\left(w_{j}^{2} \neq s_{j}^{2}\right)$.

As for $B_{1, j}$, which ensures all the coefficients pairs from $(k-1)$-th to $(j+1)$-th are the same if $\operatorname{Dec}_{\oplus}\left(B_{1, j}\right)=0$. For $j=k-1$, we have $B_{1, k-1}=\mathbf{E n c}_{\oplus}(0) \cdot \mathbf{E n c}_{\oplus}(0)=$ Enc $_{\oplus}(0+0)=$ Enc $_{\oplus}(0)$. For $j<k-1$, we have $B_{1, k-2}=$
$\operatorname{Enc}_{\oplus}\left(p_{0}^{k-1}\left(\alpha_{k-1}-\beta_{k-1}\right)\right), B_{1, k-3}=\mathbf{E n c}_{\oplus}\left[p_{0}^{k-2}\left(\alpha_{k-2}-\right.\right.$ $\left.\left.\beta_{k-2}\right)+p_{0}^{k-1}\left(\alpha_{k-1}-\beta_{k-1}\right)\right], \ldots, B_{1,0}=\mathbf{E n c}_{\oplus}\left[p_{0}\left(\alpha_{1}-\beta_{1}\right)+\right.$ $\left.\cdots+p_{0}^{k-1}\left(\alpha_{k-1}-\beta_{k-1}\right)\right]$. If $\operatorname{Dec}_{\oplus}\left(B_{1, j}\right)=0, j<k-1$, then $p_{0}^{k-1}\left(\alpha_{k-1}-\beta_{k-1}\right)+\cdots+p_{0}^{j+1}\left(\alpha_{j+1}-\beta_{j+1}\right)=0$, so $\left(\alpha_{k-1}=\beta_{k-1}\right) \wedge \cdots \wedge\left(\alpha_{j+1}=\beta_{j+1}\right)$ holds. When $\left(A_{1, j}^{1} \cdot A_{1, j}^{2}=\right.$ Enc $\left._{\oplus}(0)\right) \wedge\left(\operatorname{Dec}_{\oplus}\left(B_{1, j}\right)=0\right)$ holds, i.e., $A_{j}^{\prime}=\mathbf{E n c}_{\oplus}(0)$, there must be $\alpha>\beta$, and the $j$-th Boolean expression holds while others are False.

After the decryption, $P_{2}$ checks whether there is only one $j$ satisfying $m_{j}=0$ to decide $\alpha>\beta$. Moreover, because of the randomness of $r_{j}^{B}$ and the shuffle operation, any parties can not know the $j$-th position but $\alpha>\beta$ as desired.

\subsection{Security}

Our three-round protocol eICP described above is secure under adversary model mentioned in section 3. By Definition 3.1 , we prove security for two parties, $P_{1}$ and $P_{2}$, holding $\beta$ and $\alpha$ in base $d_{M}$, respectively. In our protocol, $F$ is a function with inputs $(\alpha, \beta)$ and output $\rho$, where $\rho=$ True if $\alpha>\beta$ and $\rho=$ False otherwise.

THEOREM 5.1. The protocol eICP (П) protects the privacy of both $P_{1}$ and $P_{2}$ under the $s R S A-S D$ Assumption.

Sketch of Proof. In order to show that $P_{2}$ learn nothing about $\beta$, we construct a valid simulator $\mathbf{S i m}_{2}$ for $P_{2}$ satisfying

$$
\operatorname{Sim}_{2}\left((\varepsilon, \alpha), \mathcal{S} \mathcal{K}_{2}, \rho, \phi_{2}\right) \approx_{c} \mathbf{V I E W}_{P_{2}}^{\Pi}\left((\beta, \alpha), \mathcal{S} \mathcal{K}_{2}, \rho, \phi_{2}\right)
$$

where $\varepsilon$ is a uniformly selected random number. To show that $P_{2}$ does not know $\beta$, we just show that $P_{2}$ can not distinguish whether $\beta$ is replaced by $\varepsilon$ or not.

Since the simulator does not know $\beta, \mathbf{S i m}_{2}$ first chooses a random $\varepsilon$ and constructs a pair $\left(C_{s}^{1}, C_{s}^{2}\right)$ by encrypting $\varepsilon$. By the semantic security of $P K E_{1}$, we have $\left(C_{s}^{1}, C_{s}^{2}\right) \approx_{c}$ $\left(C_{r}^{1}, C_{r}^{2}\right)$, where $\left(C_{r}^{1}, C_{r}^{2}\right)$ are real encryption knowing $\beta$. Then $\mathbf{S i m}_{2}$ samples $r_{2}^{1}, r_{2}^{2}, s^{1}, s^{2}$ as in the real execution to construct the quintuple. For $A_{s, j}, \mathbf{S i m}_{2}$ encrypts 0 together with another $(k-1)$ non-zero numbers randomly chosen from $M_{P K E_{2}}$ (message space) if $\rho=$ True, otherwise, $\mathbf{S i m}_{2}$ encrypts $k$ non-zero numbers. Finally, $\mathbf{S i m}_{2}$ shuffles $A_{s, j}$. We have $A_{s, j} \approx_{c} A_{r, j}$ because of the semantic security of $P K E_{2}$, so does $A_{j}$. Hence, (1) holds.

Similar to the above proof, we construct a valid simulator Sim $_{1}$ for $P_{1}$ satisfying

$$
\operatorname{Sim}_{1}\left(\beta, \mathcal{S} \mathcal{K}_{1}, \phi_{1}\right) \approx_{c} \operatorname{VIEW}_{P_{1}}^{\Pi}\left((\beta, \alpha), \mathcal{S} \mathcal{K}_{1}, \phi_{1}\right)
$$

Since the simulator holds $\beta, \mathbf{S i m}_{1}$ can compute the exchanged messages as that in the real execution. Because $\mathbf{S i m}_{1}$ does not know $\alpha$, to simulate the quintuple transferred in the second round, $\mathbf{S i m}_{1}$ uniformly chooses $2 k$ random numbers $z_{s, j}^{i}, i=1,2 ; j=k-1, \ldots, 0$ from $\left\{1, \ldots, b^{d_{M}}-1\right\}$ such that $z_{s, j}^{i} \neq 0 \bmod b$ to construct $D_{s}^{1}$ and $D_{s}^{2}$. Then $D_{s}^{i}, i=1,2$ are decrypted by $P_{1}$, in the real, $w_{j}^{1}=b^{d_{M}-\beta_{j}+\alpha_{j}}+s_{j}^{1}$, and $w_{j}^{1}$ is always not equal to $0\left(\bmod b^{d_{M}}\right)$ due to the existence of $s_{j}^{1}$, so does $w_{j}^{2}$. Hence, in the simulation case, one should ensure $z_{s, j}^{i} \neq 0 \bmod b^{d_{M}}$, since $b$ is a prime, one just chooses $z_{s, j}^{i}$ such 
that $z_{s, j}^{i} \neq 0 \bmod b$. What's more, recall that $s_{j}^{i}$ is a uniform value, so does $w_{j}^{i}$, which is consistent with $z_{s, j}^{i}$. Since $P K E_{1}$ is semantically secure, $\operatorname{Enc}_{1}\left(z_{s, j}^{i}\right)$ is indistinguishable from the real ciphertext. As for $\left(A_{2}^{1}, A_{2}^{2}, B_{2}\right)_{s}, \mathbf{S i m}_{1}$ selects $3 k$ random numbers from the message space of $P K E_{2}$ and encrypts them, a PPT algorithm can not distinguish $\left(A_{2}^{1}, A_{2}^{2}, B_{2}\right)_{s}$ with $\left(A_{2}^{1}, A_{2}^{2}, B_{2}\right)_{r}$ since $P K E_{2}$ is semantically secure. Finally Sim $_{1}$ performs the same computations in the third round as in the real, and the semantic security of $P K E_{2}$ guarantees the indistinguishability between $A_{s, j}$ and $A_{r, j}$. Hence, (2) holds.

\subsection{Extended Auction Scheme}

Here we can get an extended version of our Auction scheme by replacing ICP with eICP above, where $\mathbf{B}_{\mathbf{i}}$ plays the role of $P_{2}$ and $\mathbf{B}_{\mathbf{h}}$ act as $P_{1}$. So we have the following theorem whose proof is similar to that of Theorem 4.1.

TheOREM 5.2. Under sRSA-SD Assumption, the extended Auction scheme protects the bid privacy of all bidders. Moreover, honest bidders can redeem their deposit.

\subsection{Further Application}

Our auction scheme described above can also be used to joint bidding. Joint bidding allows some firms to collectively undertake a big project beyond any single. The logic is to take advantage of the collective size of the group. The firms need to develop a group identity and act collegially in order to win the project. To choose proper partners, the project owner need to check their financial assets which they do not like to uncover.

The project owner A first evaluates the cost of the project, say $x$, then commits to it and broadcasts the commitment onto blockchain. On the other hand, every firm $\mathbf{B}_{\mathbf{i}}$ commits to its assets $y_{i}$ and broadcasts it onto blockchain.

Afterwards, $\mathbf{A}$ runs eICP with $\mathbf{B}_{\mathbf{i}}$ to compare $x$ with $y_{i}$. If $y_{i}>x, \mathbf{A}$ then accepts $\mathbf{B}_{\mathbf{i}}$ as a candidate; Otherwise, $\mathbf{B}_{\mathbf{i}}$ can redeem his deposit by CS.Open or CS.Refund. A then selects another $\mathbf{B}_{\mathbf{i}+\mathbf{1}}$ to do further comparisons. During the whole process, everyone's privacy is protected. Specifically, they do the follows.

(1) A first evaluates the cost of the project, say $x$, and makes a commitment to $x$ and broadcasts it onto blockchain.

(2) Every firm $\mathbf{B}_{\mathbf{i}}$ commits its asset $y_{i}$ by CS, and then run eICP to compare $y_{i}$ with $x$. If $y_{i}>x, \mathbf{A}$ accepts $\mathbf{B}_{\mathbf{i}}$ as a candidate; otherwise $\mathbf{B}_{\mathbf{i}}$ can redeem his deposit by CS.Open or CS.Refund. $\mathbf{A}$ then selects another $\mathbf{B}_{\mathbf{i}+\mathbf{1}}$.

(3) Repeat step 2 until selecting all the candidates to do further considerations.

When the protocol completed, A can make the decision from all the candidates according to other considerations like work experience, etc. Here, we run eICP between $\mathbf{B}_{\mathbf{i}}$ and $\mathbf{A}$ every time, and the asset privacy of all firms is preserved. Moreover, honest firms will not lose their deposit.

\section{CONCLUSION}

In this paper, we present sequential and parallelized auction schemes via a three-round integer comparison protocol and a commitment scheme with deposit. Our schemes perform well in correctness and privacy-preserving. Moreover, we extend the comparison protocol to compare larger integers, which makes our auction schemes much more practical. These schemes can be widely used in some related topics, such as joint bidding and so on. Furthermore, in the schemes, blockchain is used to broadcast commitment and redeem deposit, as well as to submit complaint and publish the result. On the other hand, our scheme needs interaction between the bidders who obtains the comparison result and sends it to the auctioneer. It is an interesting study on how to remove the requirement of network connectivity between bidders, at the same time, the auctioneer can get the result by himself other than from the bidders.

\section{REFERENCES}

[1] Marcin Andrychowicz, Stefan Dziembowski, Daniel Malinowski, and Lukasz Mazurek. 2014. Secure multiparty computations on bitcoin. In Security and Privacy (SP), 2014 IEEE Symposium on. IEEE, 443-458.

[2] David W Archer, Dan Bogdanov, Benny Pinkas, and Pille Pullonen. 2016. Maturity and performance of programmable secure computation. IEEE security and privacy 14, 5 (2016), 48-56.

[3] Iddo Bentov and Ranjit Kumaresan. 2014. How to Use Bitcoin to Design Fair Protocols. In Cryptology Conference. 421-439.

[4] Erik-Oliver Blass and Florian Kerschbaum. 2018. Strain: A Secure Auction for Blockchains. In Computer Security, Javier Lopez, Jianying Zhou, and Miguel Soriano (Eds.). Springer International Publishing, Cham, 87-110.

[5] Felix Brandt. 2003. Fully Private Auctions in a Constant Number of Rounds. Springer Berlin Heidelberg. 223-238 pages.

[6] Rhys Carlton, Aleksander Essex, and Krzysztof Kapulkin. 2018. Threshold Properties of Prime Power Subgroups with Application to Secure Integer Comparisons. In Cryptographers Track at the RSA Conference. Springer, 137-156.

[7] Farok J. Contractor. 2002. Cooperative Strategies and Alliances in International Business. Elsevier Science,. 489-491 pages.

[8] H Galal and A Youssef. 2018. Verifiable sealed-bid auction on the ethereum blockchain. In International Conference on Financial Cryptography and Data Security, Trusted Smart Contracts Workshop. Springer.

[9] Yutao Jiao, Ping Wang, Dusit Niyato, and Kongrath Suankaewmanee. 2018. Auction Mechanisms in Cloud/Fog Computing Resource Allocation for Public Blockchain Networks. (2018).

[10] Ahmed Kosba, Andrew Miller, Elaine Shi, Zikai Wen, and Charalampos Papamanthou. 2016. Hawk: The blockchain model of cryptography and privacy-preserving smart contracts. In 2016 IEEE symposium on security and privacy (SP). IEEE, 839-858.

[11] Vijay Krishna. 2009. Auction Theory. Number 9780123745071 in Elsevier Monographs. Elsevier. https://ideas.repec.org/b/eee/ monogr/9780123745071.html

[12] Ranjit Kumaresan and Iddo Bentov. 2016. Amortizing Secure Computation with Penalties. In ACM Sigsac Conference on Computer and Communications Security. 418-429.

[13] Ranjit Kumaresan, Vinod Vaikuntanathan, and Prashant Nalini Vasudevan. 2016. Improvements to Secure Computation with Penalties. In ACM Sigsac Conference. 406-417.

[14] Yehuda Lindell, Benny Pinkas, Nigel P. Smart, and Avishay Yanai. 2015. Efficient Constant Round Multi-party Computation Combining BMR and SPDZ. Springer Berlin Heidelberg. 319-338 pages.

[15] David Cerezo Sánchez. 2018. Raziel: Private and Verifiable Smart Contracts on Blockchains. (2018). https://eprint.iacr.org/2017/ 878.pdf

[16] Berry Schoenmakers and Pim Tuyls. 2004. Practical twoparty computation based on the conditional gate. Advances in Cryptology-ASIACRYPT 2004 (2004). 